\title{
A Dynamic RWA Algorithm in a Wavelength-Routed All-Optical Network with Wavelength Converters ${ }^{1}$
}

\author{
Xiaowen Chu, Bo Li \\ \{chxw, bli\}@cs.ust.hk \\ Department of Computer Science \\ The Hong Kong University of Science and Technology
}

\author{
Zhensheng Zhang \\ zzhang@ieee.org \\ Microsoft Research Asia (visiting) \\ No. 49, Zhichun Road Haidian District, Beijing, China
}

\begin{abstract}
Existing research demonstrated that an effective Routing and Wavelength Assignment (RWA) scheme and a wavelength converter placement algorithm are the two primary vehicles for improving the blocking performance in a wavelength-routed all-optical network. However, these issues have largely been investigated separately, in particular, the RWA has seldom considered the existence of wavelength converters. In this paper, we argue perhaps for the first time, that an effective RWA algorithm needs to take into account the presence of wavelength conversion as the later is usually done at much earlier stage during the capacity planning. We proceed to show that existing dynamic RWA algorithms largely fail in the presence of wavelength conversion. We then propose a weighted least-congestion routing and first-fit wavelength assignment (WLCR-FF) RWA algorithm in conjunction with a simple heuristic wavelength converter placement algorithm called Minimum Blocking Probability First (MBPF) that considers both the distribution of free wavelengths and the lengths of each route jointly. We further introduce an analytical model that can obtain the blocking performance of the proposed WLCR routing algorithm. Using both analysis and simulation, we carry out extensive numerical studies over the typical topologies including the ring, mesh-torus, and two mesh topologies, the 14-node NSFNET and the 19-node European Optical Network (EON); we compare the performance of proposed algorithm with a wide variety of existing routing algorithms including static routing, fixed-alternate routing and least-loaded routing algorithms. The results conclusively demonstrate that the proposed WLCR-FF algorithm can achieve much better blocking performance in the environment of sparse or/and full wavelength conversion.
\end{abstract}

Index terms-- Routing and wavelength assignment, wavelength routing, wavelength converter placement

\section{INTRODUCTION}

Wavelength-routed all-optical networks are considered to be candidates for the next generation wide-area backbone networks [20]. An all-optical wavelength-routed wavelength division multiplexing (WDM) network consists of optical wavelength routing nodes interconnected by optical fiber links. A lightpath has to be established before the communication between any two routing nodes. It represents a direct optical connection between two end nodes without any intermediate electronics [5]. To establish a lightpath, it is normally required that the same wavelength should be allocated on all the links along the path. This limitation is known as the wavelength continuity constraint, which makes the wavelength-routed networks different from the traditional circuit-switched telephone networks. A sequence of lightpath requests arrives over time and each lightpath has a random

\footnotetext{
1 The work is supported in part by RGC grants under contracts AoE/E01/99 and HKUST 6196/02E.
}

holding time. These lightpaths need to be set up dynamically by determining a route across the network connecting the source to the destination and assigning a free wavelength along the path. The existing lightpaths cannot be re-routed to accommodate the new lightpath requests until they are released. So some of the lightpath requests may be blocked if there is no free wavelength along the path. One of the primary design objectives of wavelength-routed all-optical networks is to minimize the blocking probability.

Wavelength conversion can eliminate the wavelength continuity constraint and thus improve the blocking performance significantly [16]. Kovacevic and Acampora investigated the blocking performance in WDM networks with and without wavelength converters in [13]. Since the wavelength converters are still very expensive nowadays, much research work focuses on sparse wavelength conversion, in which only part of the network nodes have the capability of wavelength conversion. If all the network nodes are capable of wavelength conversion, this is referred to as full wavelength conversion. Subramaniam et al. have shown that, by using sparse wavelength conversion, a relatively small number of converters can achieve satisfactory performance [22]. The problem of wavelength converter placement is also very important. That is, given a network topology, a certain number of wavelength converters, and traffic statistics, how can the wavelength converters be placed in order to minimize the overall blocking probability? The algorithms for optimal converter placement for simple topologies, such as bus and ring, have been provided in [23]. However, optimal converter placement for more realistic topologies such as arbitrary mesh is considered to be very hard. Hence, a number of heuristic algorithms have been proposed [1] [7] [8] [10] [24].

Existing research demonstrates that an effective routing and wavelength assignment (RWA) strategy and a proper wavelength converter placement algorithm are the two primary vehicles for improving the blocking performance [6] [12] [17] [19] [25] [26]. However, these two issues have largely been investigated separately in that the existing RWA algorithms have seldom considered the presence of wavelength conversion, while the wavelength converter placement algorithms have largely assumed that a static routing and random wavelength assignment algorithm is employed. Our study in this paper is mainly motivated by the observation that the conventional dynamic routing algorithms may not work well in the environment with sparse or/and full wavelength conversion. The main reason is that the existing dynamic routing algorithms usually only take into account the distribution of free wavelengths, i.e., they usually select a route with more free wavelengths, and do not explicitly 
consider the length of routes. Evidently, with no wavelength conversion, the route with more free wavelengths usually has shorter length, as the probability of a longer route has more free wavelength is much smaller comparing to that of a shorter route. However, with the presence of wavelength converter, the above property no longer holds. Consider an example in ring networks with full wavelength conversion, the least-loaded routing (LLR) [3] algorithm actually resulted in worse performance comparing to that of the static fixedalternate routing algorithm (Further elaboration will be given in Section $\mathrm{V}$ by numerical results).

In this paper, we propose a new dynamic RWA algorithm, called weighted least-congestion routing and first-fit wavelength assignment (WLCR-FF), which considers the distribution of free wavelengths and the lengths of each route jointly. In addition, we propose an efficient heuristic converter placement algorithm called Minimum Blocking Probability First $(M B P F)$ algorithm, which is designed for our WLCR-FF RWA algorithm. Using both analysis and simulation, we carry out extensive performance studies of the proposed WLCR-FF and MBPF algorithms over a variety of topologies including ring topology, mesh-torus topology and two typical mesh topologies, the 14-node NSFNET and the 19 -node EON. The results conclusively demonstrate that the proposed WLCR-FF and MBPF algorithms can achieve much better performance than static routing, fixed-alternate routing and conventional dynamic routing algorithms, in the environment with sparse or/and full wavelength conversion. When there is no wavelength conversion, the proposed WLCR-FF can achieve similar performance as least-loading routing and first-fit (LLR-FF) algorithm.

It is not the objective of this paper to propose the best possible RWA in the presence of wavelength converters, which is very difficult and subject to further research; instead the primary objective in this paper is to present a convincing argument and evidence that RWA and wavelength converter placement need to be considered jointly. This is highlighted by the significant performance gain in terms of blocking probability observed from extensive numerical studies in using the proposed WLCR-FF algorithm. In addition, our contribution lies in the introduction of a new analytical model that can derive the performance of RWA algorithms under the presence of wavelength converters.

The rest of the paper is organized as follows. In Section II, we discuss relevant work. In Section III, we present the WLCR-FF RWA algorithm. In section IV, we consider the sparse wavelength conversion and present the MBPF algorithm for wavelength converter placement for an arbitrary mesh network that employs the WLCR-FF RWA algorithm. In section $\mathrm{V}$, we evaluate the blocking performance of the WLCR-FF algorithm in different topologies, and also we discuss the performance measures in terms of the average route length and the link utilization. Finally, Section VI concludes the paper. The analytical model of WLCR is given in the Appendix.

\section{THE RELATED WORK}

Routing and wavelength assignment (RWA) algorithms play a key role in improving the blocking performance of wavelength-routed all-optical networks. Shortest path routing strategy has been widely used in telephone network and Internet simply because it consumes less resource. Many variations of shortest path routing strategy have also been proposed and investigated in the domain of optical network. Generally these routing strategies can be classified into two categories: static routing and dynamic (or adaptive) routing. In static routing, the routes are usually determined under a prior-given traffic matrix without considering the current network state (e.g. the load distribution of each link); while in dynamic routing, the route selection is based on the current network state.

Birman introduced a reduced load approximation scheme to calculate the blocking probabilities for fixed shortest path routing in arbitrary topologies [3], which showed that the blocking probabilities grow with the number of hops much faster than for circuit-switched telephone network due to the wavelength continuity constraint. This result has also been exposed by Barry and Humblet [2]. However, the performance of fixed shortest path routing is very limited because the traffic is distributed to the links that belong to some shortest paths. These links are heavily loaded while the other links are very lightly loaded, resulting in very low fiber link utilization. To alleviate the drawback of fixed shortest path routing algorithm, Harai et al. proposed the fixedalternate routing algorithm [9] and investigated its performance by extending Birman's analytical model. The fixed-alternate routing algorithm can improve the blocking performance by introducing more routes between each pair of nodes. If there is no available wavelength on the primary route, an alternative route will be tried. Thus the traffic potentially can be distributed to more fiber links, and the overall blocking performance can be improved. A new analytical technique for the analysis of all-optical networks without wavelength conversion has been proposed in [21]. This technique is based on the inclusion-exclusion principle from combinatorics, and it can also be extended to analyze fixed-alternate routing algorithms.

The main problem of static routing strategies is that the route decision does not consider the current network state. In another word, the static routing strategies lack the capability of traffic engineering, which is very important in the dimensioning of backbone network. On the contrary, dynamic routing algorithms are good candidates for traffic engineering and they can further improve the blocking performance significantly [3] [4] [11] [14] [15]. In dynamic routing, the route decision is based on the current network state. The network state can be managed in either a distributed manner or a centralized manner. For scalability, distributed management is often preferred [18]. Least-loaded routing (LLR) is one of the early-proposed dynamic routing strategies [3]. The main idea of LLR is borrowed from telephone network in that it requires that the network is fully connected and the paths can have at most two hops. If a connection cannot be set up along the direct route, a two-hop alternate route with the largest number of free wavelengths is chosen. Birman also introduced a reduced load approximation scheme to calculate the blocking probabilities for LLR. However, there is no performance comparison between LLR and other 
routing strategies. $\mathrm{Li}$ and Somani proposed a dynamic routing algorithm named fixed-paths least-congestion routing (FPLC), based on path and neighborhood link congestion [15]. The FPLC algorithm routes a connection request on the least-congested path out of a set of pre-determined paths. The results showed that the FPLC algorithm can improve the performance significantly compared to fixed-alternate routing algorithms. However, in the presence of wavelength conversion, the conventional dynamic routing algorithms, such as LLR and FPLC algorithms, do not work well because they only take into account the distribution of free wavelengths and don't consider the route length explicitly. Recently, two dynamic routing strategies for the case of full wavelength conversion have been proposed [11] [14]. Lang et al. presented an analysis for dynamic routing in regular torus network with full wavelength conversion [14]. Hsu et al. proposed a weighted-shortest path strategy, which looks for the path that minimizes the resource cost while maintaining the traffic load among the links as balanced as possible [11]. Both works have shown the importance of re-examination of RWA problem in the presence of wavelength conversion. However, only the case of full wavelength conversion has been investigated.

\section{WLCR-FF ALGORITHM}

Routing and wavelength assignment algorithms play a key role in improving the blocking performance of wavelengthrouted networks. Dynamic routing algorithms have been shown to achieve much better blocking performance than static routing and fixed-alternate routing when the networks have no wavelength conversion. In the conventional dynamic RWA algorithms, a set of routes connecting the sourcedestination pair is searched in parallel, and the route with the maximum number of free wavelengths is selected to set up the lightpath.

In this section, we propose a new dynamic RWA algorithm that considers the distribution of free wavelengths and the lengths of each route jointly. The literature results have shown that the first-fit wavelength assignment scheme can achieve almost the same performance as the most-used wavelength assignment [27] and it is very simple for implementation. Our proposed dynamic RWA algorithm combines the best features of the weighted least-congestion routing algorithm and the first-fit wavelength assignment scheme, abbreviated as WLCR-FF algorithm.

\section{A. System Parameters and Assumptions}

In this paper, we make the following assumptions.

1. The network consists of $N$ nodes and $J$ fiber links. Each link has $W$ wavelengths that are labelled from 1 to $W$.

2. Following convention, we assume that lightpath connection requests arrive at end-to-end node pair $a$

following a Poisson distribution with rate $A^{a}$. We also assume that the connection holding times are exponentially distributed with a unit time.

3. A route $R$ is a subset of the link set $\{1,2, \ldots, J\}$. The length of route $R$ is denoted as $h(R)$.
4. Let $m_{j}$ denote the number of free wavelengths on link $j$.

5. $\left\{R_{a}^{(1)}, R_{a}^{(2)}, \ldots, R_{a}^{\left(M_{a}\right)}\right\}$ is the set of routes pre-computed for node pair $a$. These routes are required to be edge-disjoint such that the blocking events on these routes could be considered to be independent approximately.

6. The number of free wavelengths on route $R_{a}^{(t)}$ is denoted as $F\left(R_{a}^{(t)}\right)$. In the case of no wavelength conversion, $F\left(R_{a}^{(t)}\right)$ is the number of common free wavelengths on all the links of the route. In the case of full wavelength conversion, $F\left(R_{a}^{(t)}\right)$ is defined as $\min \left\{m_{j}\right\}$ where link $j$ is contained in route $R_{a}^{(t)}$. In the case of sparse wavelength conversion, let's say there are $w_{a}^{t}$ wavelength converters in route $R_{a}^{(t)}$ (excluding the two end nodes), we can divide the route into $w_{a}^{t}+1$ segments, as illustrated in Fig. 1. The $k$ th segment is denoted by $R_{a}^{(t, k)}$. The number of free wavelengths for segment $R_{a}^{(t, k)}$ is represented as $f\left(R_{a}^{(t, k)}\right)$. The number of free wavelengths of route $R_{a}^{(t)}$ is defined as the minimum value of $f\left(R_{a}^{(t, k)}\right)$ among all the segments in route $R_{a}^{(t)}$, i.e., $F\left(R_{a}^{(t)}\right)=\min \left\{f\left(R_{a}^{(t, k)}\right)\right\}$.

7. The term "offered traffic" means the traffic that arrives, and "carried traffic" means the traffic that can be actually set up successfully. $A^{a}$ is the offered traffic for node pair $a$, and $\overline{A^{a}}$ is the carried traffic for node pair $a$.

8. $B_{R_{a}^{(t)}}$ is the blocking probability of the route $R_{a}^{(t)}$.

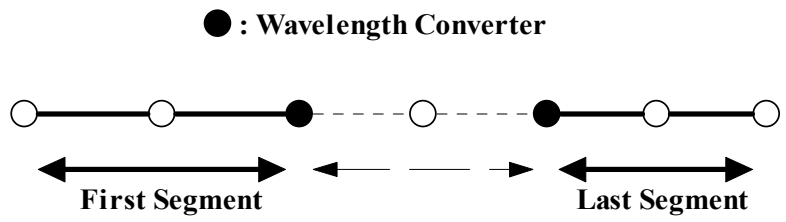

Fig. 1. A route and its segments

\section{B. Description of the Weighted Least-Congestion Routing and First-fit Wavelength Assignment Algorithm}

In the WLCR-FF RWA algorithm, a set of routes have been pre-computed for each source-destination pair, which are usually the edge-disjoint $k$-shortest paths. These routes will be re-computed if the network topology is changed. If a lightpath connection request comes to a node pair, it should make a decision to choose a route from the pre-computed set of routes, and then assign a free wavelength to the selected route. The objective of the RWA algorithm is to carry more traffic while keeping the blocking probability very low.

Let $\left\{R_{a}^{(1)}, R_{a}^{(2)}, \ldots, R_{a}^{\left(M_{a}\right)}\right\}$ denote the set of routes precomputed for node pair $a$. Upon arrival of a connection request for node pair $a$, a route has to be selected from the 
$M_{a}$ number of candidate routes. The WLCR-FF algorithm will make a route decision as follows:

We associate a weight value $W(R)$ for each candidate route. The weight function $W(R)$ is defined as:

$$
W(R)=\frac{F(R)}{\sqrt{h(R)}} .
$$

After computing all the weight values, we choose the route with the maximum weight value to setup the lightpath. If no wavelength is available on any of the routes, i.e., $F(R)=0$ for all the routes, the connection request is blocked. Once a lightpath is setup, the first-fit wavelength assignment scheme will be employed on each segment in the selected route, i.e., for each segment, the free wavelength with the smallest label will be assigned to all the links in that segment.

The selection of the weight function $W(R)$ is based on the following observation: When we make a route decision, two important factors should be considered: the number of free wavelengths and the lengths of the routes. Intuitively, the route with more free wavelengths should be selected and at the same time the length of that route should not be too long. If there is no wavelength conversion, these two factors are correlated, i.e., a shorter route is likely to have more free wavelengths than the longer routes. So the conventional dynamic RWA algorithms work very well in the networks without wavelength conversion by selecting the route with more free wavelengths. However, if the network has the capability of wavelength conversion, the correlation between the number of free wavelengths and the route lengths is weakened in the sense that a longer route is possible to have more free wavelengths than the shorter routes. Thus if we still select the route with more free wavelengths, it's possible that such routes are longer, which potentially results in a high blocking probability. In principle, the weight function should be proportional to the number of free wavelengths, and be inversely proportional to the length of the route, which is the main reason for the selection of the weighted function.

Both the analytical model and numerical algorithm to calculate the blocking probability for the WLCR routing algorithm are presented in the Appendix.

\section{HEURISTIC WAVELENGTH CONVERTER PLACEMENT ALGORITHM FOR WLCR-FF}

An exhaustive approach by enumerating all the possible ways of converter placement and choosing the best one is not efficient for large networks. In this section, we propose a heuristic algorithm of wavelength converter placement in an arbitrary mesh network that employs the WLCR-FF RWA algorithm. The algorithm places the converters one bye one. Each time we want to find a node from the candidate nodes such that if we put a converter on that node, the overall blocking probability can be decreased most significantly in a greedy fashion. The algorithm is thus called Minimum Blocking Probability First.

The MBPF algorithm works as follows:
(1) Find the routes $R_{a}^{(1)}, R_{a}^{(2)}, \ldots, R_{a}^{\left(M_{a}\right)}$ for each node pair $a$.

(2) The term "candidate node" means the node that has no converter yet. For each candidate node $v$, we first assume that a wavelength converter has been placed at that node, and then we can calculate the corresponding overall blocking probability using the analytical model presented in the Appendix. After the calculation of all candidate nodes, we place a wavelength converter at the node that can result in the minimum overall blocking probability.

(3) If there are still wavelength converters left, go to Step (2).

The MBPF algorithm will use the numerical algorithm (presented in the Appendix to calculate the blocking probability) $\mathrm{O}(M N)$ times. This is very efficient compared to the exhaust searching of all the $\left(\begin{array}{l}N \\ M\end{array}\right)$ combinations of converter placement schemes.

\section{NUMERICAL RESULTS AND ANALYSIS}

Extensive simulations have been carried out to investigate the performance of the proposed WLCR-FF algorithm over an 8-node ring topology (Fig. 2(a)), 25-node mesh-torus topology (Fig. 2(b)), 14-node NSFNET topology (Fig. 2(c)) and 19-node EON topology (Fig. 2(d)). The lightpath connection requests arrive to the network following a Poisson process, and the connection holding time is exponentially distributed. We assume that all the source-destination node pairs have the same traffic load in Erlangs. Each fiber link is assumed to carry 40 wavelength channels. In the simulations, we provide two edge-disjoint shortest paths for each sourcedestination pair. The two routes are edge-disjoint so that the blocking events on the two routes can be considered to be independent. It is also good for fault tolerance. If one route fails, the connection can be rerouted to another route. For each topology, we compare the performance of the WLCR-FF algorithm to the shortest path routing (SP-FF), fixed-alternate routing (FA-FF) and least-loaded routing (LLR-FF) algorithms under three different environments: no wavelength conversion, sparse wavelength conversion and full wavelength conversion. In the case of sparse wavelength conversion, the proposed MBPF converter placement algorithm is employed to place a limited number of wavelength converters into the network.

\section{A. Blocking Performance Analysis of the Ring Topology}

Fig. 3(a) depicts the blocking performance of different RWA algorithms in an 8-node ring network without wavelength conversion. We can observe that the FA-FF, LLR-FF and WLCR-FF algorithms work much better than the SP-FF algorithm. This can be explained as follows: when the traffic load is low, the main reason of a blocking event is that there is no common free wavelength among the links along the route. When we provide two candidate routes for a node pair, the blocking events of these two routes can be considered to be independent. Hence the blocking probability can be decreased a lot. Another observation is that the performance of WLCR-FF is very close to that of the LLR- 


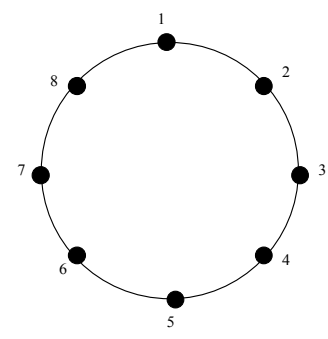

(a) An 8-node ring network

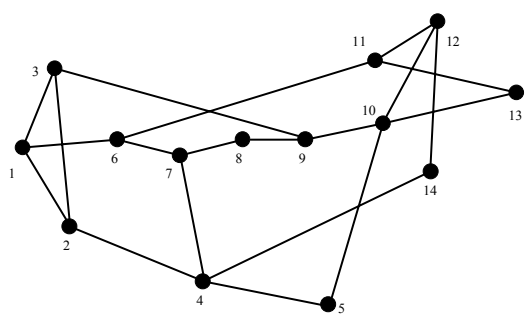

(c) 14-node NSFNET network

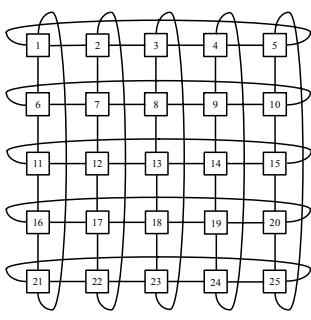

(b) A 25-node mesh-torus network

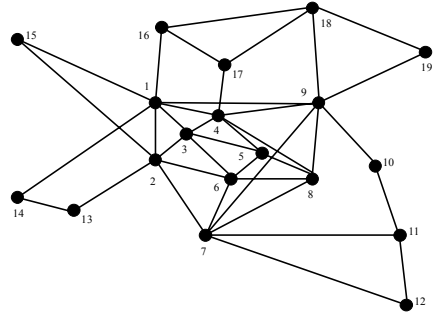

(d) 19-node European Optical Network (EON)

Fig. 2. Network Topologies

FF, which is better than the FA-FF algorithm. Dynamic RWA algorithms can improve the blocking performance because more wavelengths are left free for future connections.

Fig. 3(b) shows the network blocking probability versus the total traffic load when there are 4 wavelength converters in the ring network. According to the MBPF converter placement algorithm, these 4 converters are placed at nodes $(1,3,5,7)$. An important observation is that the blocking probability of the LLR-FF algorithm increases rapidly when the traffic load increases. The performance of the LLR-FF algorithm is even worse than that of the FA-FF algorithm. However, the WLCR-FF algorithm can still achieve better performance than the FA-FF algorithm. The drawback of the LLR-FF algorithm in the environment of sparse wavelength conversion is that, they make a route decision based on the information of free wavelengths only and they don't consider the length of each route. For most node pairs in a ring topology, one route is very short and another route is very long. The LLR-FF algorithm is likely to use too many long routes and thus consume too many resources. On the opposite, the WLCR-FF algorithm takes into account the length of each route and avoids using too many long routes. Thus the WLCR-FF can achieve the best blocking performance.

The performances of different RWA algorithms in the case of full conversion are shown in Fig. 3(c). In the full conversion network, there is no wavelength continuity constraint. For the same reason, the LLR-FF algorithm uses too many long routes and increases the blocking probability dramatically. We can observe that the performance of LLRFF algorithm is worse than the SP-FF algorithm when the total traffic load is beyond 100 Erlangs. The WLCR-FF algorithm works very well under full wavelength conversion.

If we compare the three figures in Fig. 3, we can observe that wavelength conversion doesn't help much in ring topology. This result is consistent with the conclusion in [13].
Another observation is that the performance of sparse wavelength conversion with $\mathrm{MBPF}$ wavelength converter placement algorithm is very close to the performance of full wavelength conversion in the ring topology.

\section{B. Blocking Performance Analysis of the Mesh-Torus Topology}

The performances of different RWA algorithms in meshtorus network in the environments of no wavelength conversion, sparse wavelength conversion and full wavelength conversion are depicted in Fig. 4. We omit the curve of SP-FF algorithm in these three figures, simply because that in mesh-torus networks, the blocking probability of SP-FF algorithms is too large compared to the FA-FF, LLR-FF and WLCR-FF algorithms.

From all these three figures, we can observe that both LLR-FF and WLCR-FF algorithms can improve the blocking performance significantly compared to the FA-FF algorithm. Mesh-torus network is much denser than the ring network. When the FA-FF algorithm is used, most of the traffic will be distributed to the shortest route between each pair of nodes, resulting that some links are seldom utilized. Dynamic RWA algorithms can distribute the traffic more evenly to all the links, and more free wavelengths are left for future connections. Thus they can decrease the blocking probability significantly.

From Fig. 4(b) and Fig. 4(c), we can also observe that the WLCR-FF algorithm can achieve better blocking performance than the LLR-FF algorithm in the cases of sparse conversion and full conversion. In the case of sparse conversion, we investigate the average performance of all the possible wavelength converter placement schemes besides the MBPF scheme. We can see that the blocking probability can be decreased $15-25 \%$ if the WLCR-FF algorithm is used instead of the LLR-FF algorithm. This is because the WLCR- 


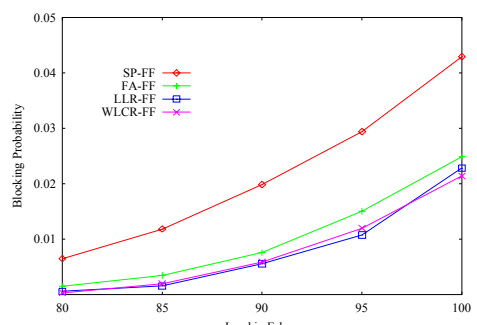

(a) No Wavelength Conversion

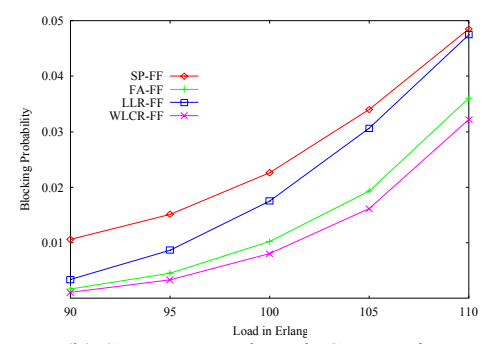

(b) Sparse Wavelength Conversion

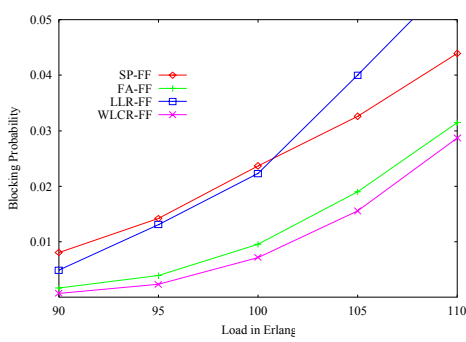

(c) Full Wavelength Conversion

Fig. 3. Blocking probability versus traffic load in 8-node Ring network

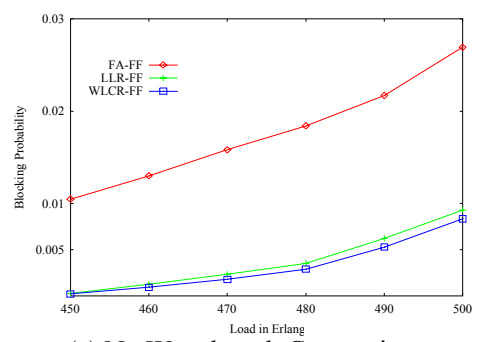

(a) No Wavelength Conversion

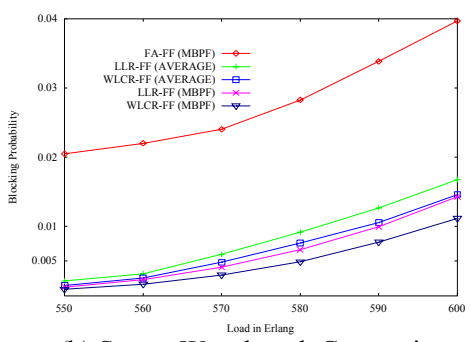

(b) Sparse Wavelength Conversion

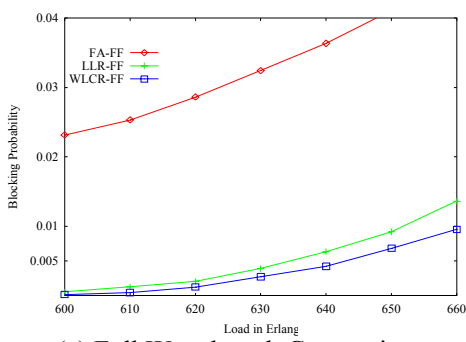

(c) Full Wavelength Conversion

Fig. 4. Blocking probability versus traffic load in 25-node Mesh-torus network

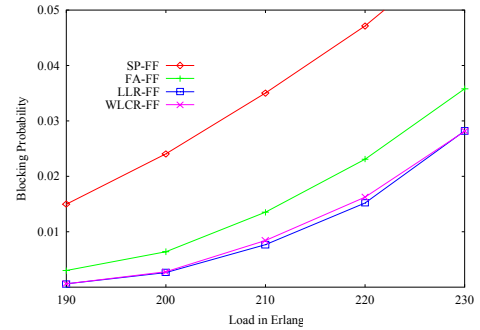

(a) No Wavelength Conversion

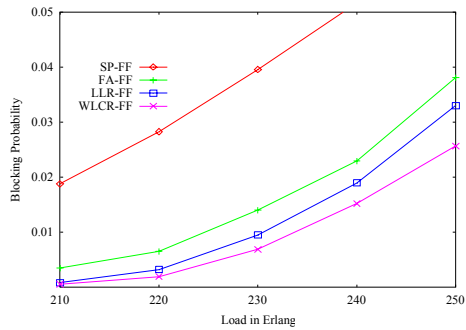

(b) Sparse Wavelength Conversion

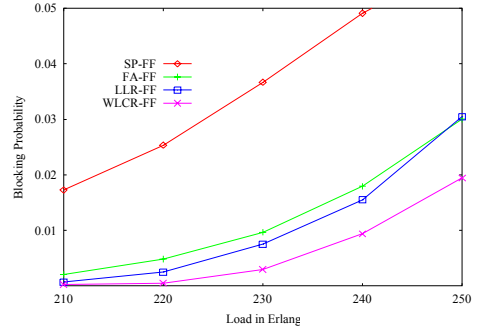

(c) Full Wavelength Conversion

Fig. 5. Blocking probability versus traffic load in 14-node NSFNET

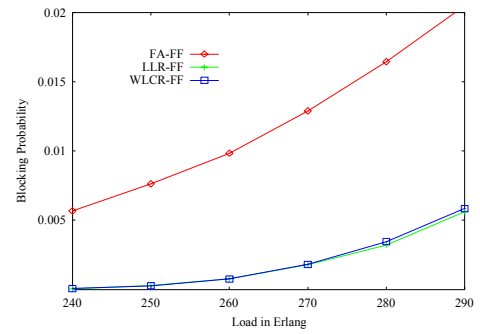

(a) No Wavelength Conversion

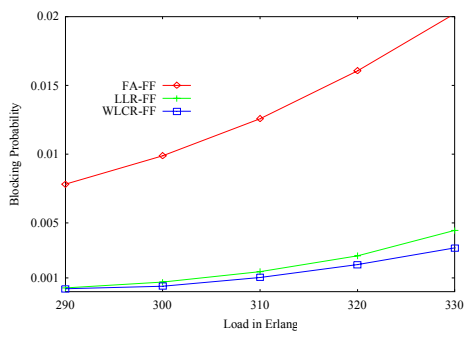

(b) Sparse Wavelength Conversion

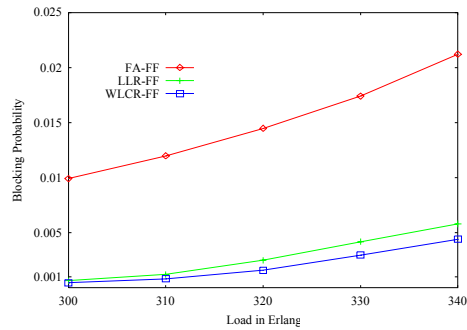

(c) Full Wavelength Conversion

Fig. 6. Blocking probability versus traffic load in 19-node EON

FF algorithm makes a better trade-off between the number of free wavelengths and the lengths of the routes. And it shows that the MBPF algorithm performs much better than the average performance over all the placement schemes.

Another important result is that wavelength conversion is very helpful in mesh-torus networks. To guarantee a blocking probability less than $1 \%$, the 25 -node mesh-torus network can carry a total traffic of 500 Erlangs without wavelength conversion. If we have 10 wavelength converters, the carried traffic can be 600 Erlangs. With full wavelength conversion, the network can carry a total traffic of 660 Erlangs. 


\section{Blocking Performance Analysis of the NSFNET Topology}

Fig. 5(a) depicts the blocking probability versus the total traffic load in NSFNET without wavelength conversion. We can see that the FA-FF algorithm works much better than the SP-FF algorithm. And the LLR-FF and WLCR-FF algorithms further improve the blocking performance.

In sparse wavelength conversion, we place 5 wavelength converters at nodes $(3,4,6,10,12)$ according to the MBPF algorithm. From Fig. 5(b), the performance of LLR-FF algorithm is better than the FA-FF algorithm. The WLCR-FF algorithm further decreases the blocking probability.

Fig. 5(c) shows the blocking performances in the environment of full wavelength conversion. The LLR-FF algorithm doesn't work well in this case. With the increase of traffic load, the blocking probability of LLR-FF algorithm is very close to or even beyond that of FA-FF algorithm. On the contrary, the WLCR-FF algorithm has a much lower blocking probability compared to both FA-FF and LLR-FF algorithms.

\section{Blocking Performance Analysis of the EON Topology}

The performance in the EON topology is similar to the mesh-torus topology. The main reason is that they are both "dense" network. The performance of SP-FF algorithm in the EON topology is also very poor and we omit the related results here. Fig. 6(a) depicts the blocking probability versus the total traffic load in EON without wavelength conversion. We can see that the LLR-FF and WLCR-FF algorithms perform much better than the FA-FF algorithm.

In sparse wavelength conversion, we place 7 wavelength converters at nodes $(1,2,4,7,9,11,18)$ according to the MBPF algorithm. From Fig. 6(b), the performance of LLR-FF algorithm is better than the FA-FF algorithm. The WLCR-FF algorithm further decreases the blocking probability. Fig. 6(c) shows the blocking performances in the environment of full wavelength conversion. If we compare Fig. 6(b) and Fig. 6(c), we find that the performance of sparse wavelength conversion is very close to that of full conversion.

\section{E. Analysis of the average route length and link utilization}

The average route lengths of different RWA algorithms in the Ring and NSFNET topologies have been shown in Table 1 and 2 respectively. First of all, the SP routing algorithm always has the shortest average route lengths. Secondly, the average route lengths of FA routing algorithm are a little longer than those of SP. The reason is that the SP routing algorithm never considers the non-shortest routes, while FA routing algorithm will try the longer alternate route if the shortest route fails. Thirdly, dynamic routing algorithms, such as the LLR and WLCR routing algorithms, result in further longer average route lengths compared to FA routing algorithm.

When there is no wavelength conversion, the differences of the average route lengths of the four routing algorithms are very minor. This is because with no wavelength conversion, the route with more free wavelengths usually has shorter length, as the probability that a longer route has more free wavelength is smaller comparing to that of a shorter route. So without wavelength conversion, for both LLR and WLCR algorithms, the probability that the alternate route is chosen is very small. However, in the case of sparse wavelength conversion and especially full wavelength conversion, the probability that the alternate routes are chosen is very high, thus the average route lengths of LLR routing algorithm are much longer than that of the FA algorithm. The WLCR algorithm makes a good compromise between FA and LLR. Since the WLCR algorithm considers the route length explicitly in the route decision, the resulted average route lengths are only a little longer than those of the FA algorithm. This makes the WLCR algorithm consume less link resources compared to LLR algorithm and thus improve the overall blocking performance.

TABLE 1. Average route length of the Ring network

\begin{tabular}{l|ccc}
\hline & No Conversion & Sparse Conversion & Full conversion \\
\hline SP & 2.23 & 2.27 & 2.28 \\
FA & 2.29 & 2.33 & 2.35 \\
LLR & 2.31 & 2.56 & 2.69 \\
WLCR & 2.30 & 2.39 & 2.39 \\
\hline
\end{tabular}

TABLE 2. Average route length of the NSFNET

\begin{tabular}{l|ccc}
\hline & No Conversion & Sparse Conversion & Full Conversion \\
\hline SP & 2.18 & 2.18 & 2.18 \\
FA & 2.19 & 2.20 & 2.20 \\
LLR & 2.28 & 2.42 & 2.54 \\
WLCR & 2.24 & 2.28 & 2.29 \\
\hline
\end{tabular}

The advantage of dynamic routing algorithms is that they can distribute the traffic to more links and thus utilize the links more efficiently. We use $u_{j}$ to denote the utilization ratio of link $j$. It is defined as

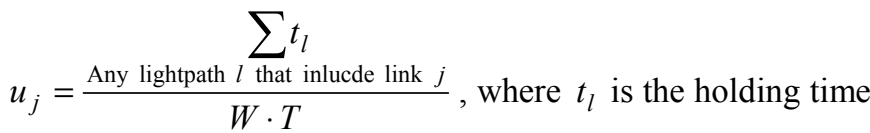
of lightpath $l, W$ is the number of wavelengths and $T$ is the total simulation time. The average link utilization ratios versus the traffic load with different wavelength conversion capabilities have been depicted in Fig. 7 and Fig. 8, for 8node ring network and 14-node NSFNET respectively. From both figures, we observe that with the growth of traffic load, the link utilization also increases. The LLR algorithm always has the highest link utilization. The WLCR algorithm also has higher link utilization than FA and SP algorithms. This is because dynamic routing algorithms can distribute the traffic to some light-loaded links. SP algorithm has the lowest link utilization because there is only one route provided for each pair nodes. FA algorithm does a better job by providing some alternate routes. However, these alternate routes are seldom used because the primary route is always considered first. We should notice that the link utilization is correlated with the average route length. Long average route length naturally results in high link utilization. This is exactly the case of LLR algorithm and it can explain why the blocking performance of LLR is worse than others in the presence of wavelength conversion, although it has the highest link utilization ratio. Another important observation is that in order to guarantee a low blocking probability, the link utilization ratio is always very low. For example, in the 8-node ring topology, without wavelength conversion, to guarantee a blocking probability of 


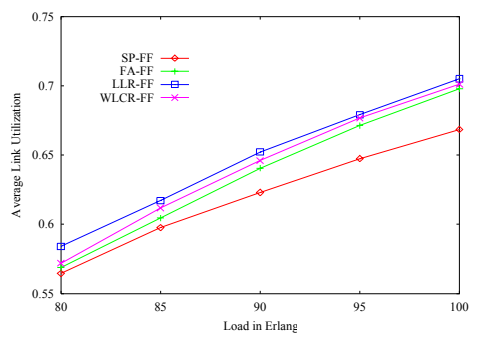

(a) No Wavelength Conversion

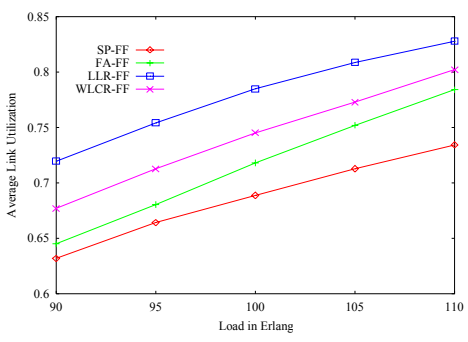

(b) Sparse Wavelength Conversion

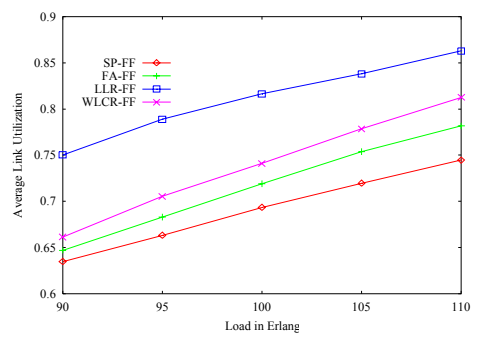

(c) Full Wavelength Conversion

Fig. 7. Average link utilization versus traffic load in 8-node Ring network

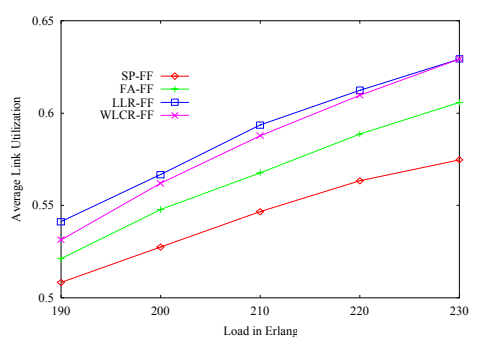

(a) No Wavelength Conversion

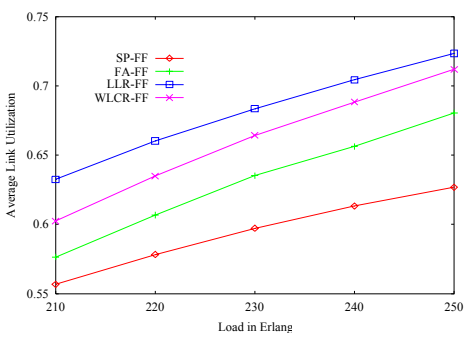

(b) Sparse Wavelength Conversion

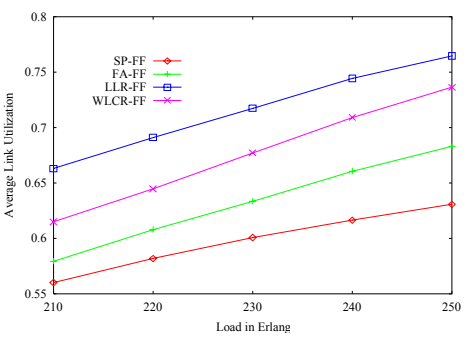

(c) Full Wavelength Conversion

Fig. 8. Average link utilization versus traffic load in 14-node NSFNET

$2 \%$, the link utilization for SP algorithm is only $62 \%$ (the traffic can only be 90 Erlangs), and the link utilization for FA, LLR and WLCR algorithms (the traffic can be 100 Erlangs) can reach $70 \%$.

In summary, the LLR algorithm has high link utilization, however, its average route length is too long; the FA algorithm has a short average route length, however, its link utilization is too low. The WLCR algorithm makes a good trade-off between the average route length and the link utilization. It can have high link utilization and keep the average route length at an acceptable level at the same time, so the overall blocking performance of the WLCR algorithm is better than others in the presence of wavelength conversion.

\section{CONCLUSIONS}

In this paper, we have examined the dynamic RWA problem in the presence of wavelength conversion. We proposed a new dynamic RWA algorithm, WLCR-FF algorithm, in wavelength-routed all-optical networks. The WLCR-FF algorithm takes into account the distribution of free wavelengths and the lengths of each route jointly when it makes a route decision. An approximate analytical model has been introduced. Furthermore, we proposed a heuristic MBPF algorithm to solve the problem of wavelength converter placement, for the case of sparse wavelength conversion. The results demonstrated that the WLCR-FF algorithm could improve the blocking performance significantly compared to conventional dynamic RWA algorithm in the environment of sparse or/and full wavelength conversion. A detailed analysis in terms of average route length and link utilization has also been presented.

\section{APPENDIX}

\section{A. Analytical Model for WLCR Routing Algorithm}

Our analytical model consists of routing analysis and path-blocking analysis. The routing analysis consists of a set of equations that determine link-offered traffic from the pathblocking probabilities. The path-blocking analysis consists of a set of equations that determine the path-blocking probabilities from the link-offered traffic. This set of fixedpoint non-linear equations can be solved by iterative substitutions.

To simplify the notations in the analysis, we assume that for each node pair $a$, only two routes are provided, denoted by $R_{a}^{(1)}$ and $R_{a}^{(2)}$. We also assume that $h\left(R_{a}^{(1)}\right) \leq h\left(R_{a}^{(2)}\right)$. This apparently can be easily extended to the case with more than two routes.

The overall blocking probability $P$ is the ratio of the blocked traffic to the offered traffic. That is,

$$
P=\frac{\sum_{a}\left(A^{a}-\overline{A^{a}}\right)}{\sum_{a} A^{a}} .
$$

The connection of node pair $a$ will be blocked only if the connection will be blocked on both candidate routes. Since the blocking events of the two routes are considered to be independent, we can have

$\overline{A^{a}}=A^{a}\left(1-\prod_{t=1}^{2} B_{R_{a}^{(t)}}\right)$. 
To obtain the steady-state probability of the number of available wavelengths on each link, we use the reduced load approximation method presented in [3]. Let $X_{j}$ denote the random variable representing for the number of free wavelengths on link $j$. We assume that the random variables $X_{j}, j \in\{1, \ldots, J\}$ are independent, and the call requests arrive at link $j$ following a Poisson distribution with rate $\alpha_{j}$. Let $q_{j}\left(m_{j}\right)$ denote the probability that $m_{j}$ wavelengths are free on link $j$. We can derive

$q_{j}\left(m_{j}\right)=P\left(X_{j}=m_{j}\right)=\frac{\prod_{i=1}^{m_{j}}(W-i+1)}{\alpha_{j}^{m_{j}}} P\left(X_{j}=0\right)$,

where

$q_{j}(0)=P\left(X_{j}=0\right)=\left[1+\sum_{m_{j}=1}^{W} \frac{\prod_{i=1}^{m_{j}}(W-i+1)}{\alpha_{j}^{m_{j}}}\right]^{-1}$.

The traffic carried on link $j$ is the sum of the carried traffic of all the routes that contain link $j$. Let $P_{a}^{(1)}$ and $P_{a}^{(2)}$ be the probabilities that a call for a node pair $a$ is set up on the first and second route respectively. Following the assumption made in [13], we can have

$\alpha_{j}\left(1-q_{j}(0)\right)=\sum_{a} A^{a}\left(P_{a}^{(1)} \beta\left(j, R_{a}^{(1)}\right)+P_{a}^{(2)} \beta\left(j, R_{a}^{(2)}\right)\right)$,

where $\beta(j, R)$ is the link-route incidence matrix defined as $\beta(j, R)=\left\{\begin{array}{l}1, j \in R \\ 0, j \notin R\end{array}\right.$.

We introduce $u_{i}\left(R_{a}^{(t, k)}\right)$ to represent the probability that $i$ wavelengths are available on segment $R_{a}^{(t, k)}$. We also introduce $u_{i}\left(m_{j} ; R_{a}^{(t, k)}\right)$ to represent the probability that when $m_{j}$ wavelengths are available on link $j, i$ wavelengths are available on segment $R_{a}^{(t, k)}$ that includes link $j$. It is easy to see that

$u_{i}\left(R_{a}^{(t, k)}\right)=\sum_{m_{j}=i}^{W} q_{j}\left(m_{j}\right) u_{i}\left(m_{j} ; R_{a}^{(t, k)}\right)$.

A route can be setup if each segment of that route has its own available wavelengths. With an approximate assumption that the blocking events of all the segments are independent, we can derive the blocking probability of any route $R_{a}^{(t)}$ as

$B_{R_{a}^{(t)}}=1-\prod_{k=1}^{w_{a}^{t}+1}\left[1-u_{0}\left(R_{a}^{(t, k)}\right)\right]$.

To determine $P_{a}^{(1)}$ and $P_{a}^{(2)}$, we need to introduce another two notation: Let $Q_{R_{a}^{(1)}}(i)$ and $Q_{R_{a}^{(2)}}(i)$ be the probabilities that $i$ wavelengths are available on route $R_{a}^{(1)}$ and $R_{a}^{(2)}$ respectively, i.e., $Q_{R_{a}^{(1)}}(i)=\operatorname{Pr}\left(F\left(R_{a}^{(1)}\right)=i\right)$ and $Q_{R_{a}^{(2)}}(i)=\operatorname{Pr}\left(F\left(R_{a}^{(2)}\right)=i\right)$. Therefore, for both $t=1$ and $t=2$, we have

$$
\begin{aligned}
& Q_{R_{a}^{(t)}}(i)=\operatorname{Pr}\left(F\left(R_{a}^{(t)}\right)=i\right) \\
& =\operatorname{Pr}\left(\min _{k \in\left\{1, \ldots, w_{a}^{(t)}+1\right\}}\left(f\left(R_{a}^{(t, k)}\right)\right)=i\right) \\
& =\sum_{m=1}^{w_{a}^{(t)}+1}\left[\prod_{j=1}^{m-1}\left(\sum_{k=i+1}^{W} u_{k}\left(R_{a}^{(t, j)}\right)\right) \cdot u_{i}\left(R_{a}^{(t, m)}\right) \cdot \prod_{j=m+1}^{w_{a}^{(t)}+1}\left(\sum_{k=i}^{W} u_{k}\left(R_{a}^{(t, j)}\right)\right)\right]
\end{aligned}
$$

According to the WLCR routing algorithm, we can have

$$
P_{a}^{(1)}=\sum_{i=1}^{W}\left[Q_{R_{a}^{(1)}}(i) \sum_{j=0}^{\phi(i)} Q_{R_{a}^{(2)}}(j)\right], \phi(i)=\left\lfloor\sqrt{\frac{h\left(R_{a}^{(2)}\right)}{h\left(R_{a}^{(1)}\right)}} \cdot i\right\rfloor,
$$

and

$$
P_{a}^{(2)}=\sum_{i=1}^{W}\left[Q_{R_{a}^{(2)}}(i) \sum_{j=0}^{\theta(i)} Q_{R_{a}^{(1)}}(j)\right], \theta(i)=\left\lfloor\sqrt{\frac{h\left(R_{a}^{(1)}\right)}{h\left(R_{a}^{(2)}\right)}} \cdot i\right\rfloor .
$$

By letting the link set of segment $R_{a}^{(t, k)}$ be $\left\{j, j_{1}, j_{2}, \cdots, j_{h\left(R_{a}^{(t, k)}\right)-1}\right\}$, the probability $u_{i}\left(m_{j} ; R_{a}^{(t, k)}\right)$ is given by the following equation if we use $h$ to denote $h\left(R_{a}^{(t, k)}\right)$, i.e., the length of the segment:

$$
\begin{aligned}
& u_{i}\left(m_{j} ; R_{a}^{(t, k)}\right)=\sum_{m_{j_{1}}=0}^{W} \sum_{m_{j_{2}}=0}^{W} \cdots \sum_{m_{j_{h-1}}=0}^{W} \\
& \left\{\prod_{l=1}^{h-1} q_{j_{l}}\left(m_{j_{l}}\right) \times p_{i}^{h}\left(m_{j}, m_{j_{1}}, \cdots, m_{j_{h-1}}\right)\right\},
\end{aligned}
$$

where $p_{i}^{n}(\cdot)$ denotes the probability that there exist $i$ available wavelengths on the $n$-hop segment, given the number of free wavelengths of all its links. It can be determined by the following recursive relation:

$$
\begin{gathered}
p_{i}^{n}\left(m_{j_{1}}, \cdots, m_{j_{n}}\right)=\sum_{k=0}^{W} p_{i}^{2}\left(k, m_{j_{n}}\right) p_{k}^{n-1}\left(m_{j_{1}}, \cdots, m_{j_{n-1}}\right), \\
p_{i}^{2}(x, y)=\left\{\begin{array}{cc}
\beta(x, y, i), & x \geq y \geq i, x+y-i \leq W, \\
\beta(y, x, i), & 1 \leq x, y \leq W \\
0, & 1 \leq x, y \leq W
\end{array}\right. \\
\hline
\end{gathered}
$$

The conditional probability $\beta(x, y, i)$ is the probability that there exist $i$ available wavelengths under the condition 
that $x$ and $y$ wavelengths are available on successive two links. From [3], $\beta(x, y, i)$ is given by

$$
\beta(x, y, i)=\left(\begin{array}{l}
y \\
i
\end{array}\right) \cdot\left(\prod_{k=1}^{i} \frac{x-k+1}{W-k+1}\right) \cdot\left(\prod_{k=1}^{y-i} \frac{W-x-k+1}{W-i-k+1}\right) .
$$

\section{B. Numerical Algorithm}

In summary, we can determine the overall blocking probability as follows:

(1) Initialize $B_{R}$ as 0 for all routes. Initialize $q_{j}(0)$ as 0 for all links. Initialize $P_{a}^{(1)}$ and $P_{a}^{(2)}$ as $1 / 2$.

(2) Calculate $\alpha_{j}$ using Eq. (5) for all links. Calculate $q_{j}(m)$ using Eq. (3) and Eq. (4) for all links.

(3) Calculate $u_{i}\left(m_{j} ; R_{a}^{(t, k)}\right)$ and $u_{i}\left(R_{a}^{(t, k)}\right)$ for all the segments using Eq. (6) and Eq. (11) - Eq. (14). Then calculate $P_{a}^{(1)}$ and $P_{a}^{(2)}$ using Eq. (8) - Eq. (10).

(4) Calculate $B_{R}$ for all routes using Eq. (7). If new values of $B_{R}$ are converged to the older ones ${ }^{2}$, the iteration is terminated and we can go to Step (5). Otherwise go to Step (2) for next iteration.

(5) Finally, calculate the overall blocking probability using Eq. (1) and Eq. (2).

\section{REFERENCES}

[1] S. Arora and S. Subramaniam, "Converter Placement in Wavelength Routing Mesh Topologies", in Proc. of IEEE ICC 2000, pages 12821288, June 2000

[2] R. A. Barry and P. A. Humblet, "Models of Blocking Probability in AllOptical Networks with and without Wavelength Changers," IEEE Journal on Selected Areas in Communications, Vol. 14, No. 5, pages 858-867, June 1996.

[3] A. Birman, "Computing Approximate Blocking Probabilities for a Class of All-Optical Networks," IEEE Journal on Selected Areas in Communications, Vol. 14, No. 5, pages 852-857, June 1996.

[4] K. Chan and T. P. Yum, "Analysis of Least Congested Path Routing in WDM Lightwave Networks," in Proc. of IEEE INFOCOM '94, pages 962-969, 1994

[5] I. Chlamtac, A. Ganz, and G. Karmi, "Lightpath Communications: An Approach to High Bandwidth Optical WAN's," IEEE Transactions on Communications, Vol. 40, No. 7, pages 1171-1182, July 1992.

[6] I. Chlamtac, A. Farago, and T. Zhang, "Lightpath (Wavelength) Routing in Large WDM Networks," IEEE Journal on Selected Areas in Communications, Vol. 14, No. 5, pages 909-913, June 1996.

[7] X.-W. Chu and B. Li, "Wavelength Converter Placement under the Fixed-Alternate Routing Algorithm in Wavelength-Routed All-Optical Networks," accepted and to appear in SPIE/Kluwer Optical Networks Magazine.

[8] X.-W. Chu and B. Li, "Wavelength Converter Placement under Different RWA Algorithms in Wavelength-Routed All-Optical Networks," accepted and to appear in IEEE Transactions on Communications.

[9] H. Harai, M. Murata, and H. Miyahara, "Performance of Alternate Routing Methods in All-Optical Switching Networks," in Proc. of IEEE INFOCOM '97, pages 517-525, April 1997.

\footnotetext{
2 The convergence here means that the difference between the new value and old value is less than some pre-defined small value.
}

[10] H. Harai, M. Murata, and H. Miyahara, "Heuristic Algorithms of Allocation of Wavelength Convertible Nodes and Routing Coordination in All-Optical Networks", IEEE/OSA Journal of Lightwave Technology, pages 535-545, April 1999

[11] C.-F. Hsu, T.-L. Liu, and N.-F. Huang, "On Adaptive Routing in Wavelength-Routed Networks", SPIE/Kluwer Optical Networks Magazine, Vol. 3, No. 1, pages 15-24, January 2002.

[12] E. Karasan and E. Ayanoglu, "Effects of Wavelength Routing and Selection Algorithms on Wavelength Conversion Gain in WDM Optical Networks," IEEE/ACM Transactions on Networking, Vol. 6, No. 2 , pages 186-196, April 1998.

[13] M. Kovacevic and A. Acampora, "Benefits of Wavelength Translation in All-Optical Clear-Channel Networks," IEEE Journal on Selected Areas in Communications, Vol. 14, No. 5, pages 868-880, June 1996.

[14] J. P. Lang, V. Sharma, and E. A. Varvarigos, "An Analysis of Oblivious and Adaptive Routing in Optical Networks with Wavelength Translation", IEEE/ACM Transactions on networking, Vol. 9, No. 4, pages 503-517, August 2001.

[15] L. Li and A. K. Somani, "Dynamic Wavelength Routing Using Congestion and Neighborhood Information," IEEE/ACM Transactions on networking, Vol. 7, No. 5, pages 779-786, October 1999.

[16] B. Ramamurthy and B. Mukherjee, "Wavelength Conversion in WDM Networking," IEEE Journal on Selected Areas in Communications, Vol. 16, No. 7, pages 1061-1073, September 1998.

[17] S. Ramamurthy and B. Mukherjee, "Fixed-Alternate Routing and Wavelength Conversion in Wavelength-Routed Optical Networks," IEEE GLOBECOM, pages 2295-2302, November 1998.

[18] R. Ramaswami and A. Segall, "Distributed Network Control for Optical Networks," IEEE Transactions on Communications, Vol. 5, No. 6, pages 936-943, December 1997.

[19] R. Ramaswami and K. Sivarajan, "Routing and Wavelength Assignment in All-Optical Networks," IEEE/ACM Transactions on Networking, Vol. 3, No. 5, pages 489-500, October 1995.

[20] R. Ramaswami and K. Sivarajan, Optical Networks: A Practical Perspective. Morgan Kaufmann Publishers, San Francisco, CA, 1998.

[21] A. Sridharan and K. N. Sivarajan, "Blocking in All-Optical Networks," in Proc. of IEEE INFOCOM '00, pages 990-999, March 2000.

[22] S. Subramaniam, M. Azizoglu, and A. K. Somani, "All-Optical Networks with Sparse Wavelength Conversion," IEEE/ACM Transactions on Networking, Vol. 4, No. 4, pages 544-557, August 1996.

[23] S. Subramaniam, M. Azizoglu, and A. K. Somani, "On Optimal Converter Placement in Wavelength-Routed Networks," IEEE/ACM Transactions on networking, Vol. 7, No. 5, pages 754-767, October 1999.

[24] K. R. Venugopal, M. Shivakumar, and P. S. Kumar, "A Heuristic for Placement of Limited Range Wavelength Converters in All-Optical Networks," in Proc. of IEEE INFOCOM '99, pages 908-915, March 1999.

[25] H. Zang and B. Mukherjee, "A Review of Routing and Wavelength Assignment Approaches for Wavelength-Routed Optical WDM Networks," SPIE/Kluwer Optical Networks Magazine, Vol. 1, No. 1, pages 47-60, January 2000.

[26] Z. Zhang and A. S. Acampora, "A Heuristic Wavelength Assignment Algorithm for Multihop WDM Networks with Wavelength Routing and Wavelength Re-use," IEEE/ACM Transactions on Networking, Vol. 3, No. 3, pages 281-288, June 1995.

[27] Y. Zhu, G. N. Rouskas, and H. G. Perros, "A Comparison of Allocation Policies in Wavelength Routing Networks," Photonic Networks Communication Journal, Vol. 2, No. 3, pages 265-293, August 2000. 